\title{
Double-Negative Prostate Carcinoma
}

National Cancer Institute

\section{Source}

National Cancer Institute. Double-Negative Prostate Carcinoma. NCI Thesaurus. Code C161609.

A metastatic, castration-resistant prostate carcinoma that is composed of fibroblast growth factor-driven malignant cells. 